\title{
Suppressing backscattering of helical edge modes with a spin bath
}

\author{
Andrey A. Bagrov,,${ }^{1,2, *}$ Francisco Guinea, ${ }^{3,4, \dagger}$ and Mikhail I. Katsnelson ${ }^{1, \dagger}$ \\ ${ }^{1}$ Institute for Molecules and Materials, Radboud University, Heijndaalseweg 135, 6525 AJ Nijmegen, The Netherlands \\ ${ }^{2}$ Department of Physics and Astronomy, Uppsala University, Box 516, SE-75120 Uppsala, Sweden \\ ${ }^{3}$ IMDEA-Nanoscience, Calle de Faraday 9, E-28049 Madrid, Spain \\ ${ }^{4}$ School of Physics and Astronomy, University of Manchester, Manchester M13 9PY, United Kingdom
}

(Received 11 July 2018; revised manuscript received 17 January 2019; published 22 November 2019)

\begin{abstract}
We address the question of stability of protected helical edge states at the boundary of two-dimensional topological insulators upon interactions with the external bath. We study how backscattering amplitude changes when different interaction channels between the system and the environment are present. Drawing an analogy with the concept of pointer states in theory of open quantum systems, we demonstrate that in a certain regime the interplay between the Coulomb and the spin-spin interactions can make the backscattering process strictly irrelevant, and the helical modes become robust states with a well-defined momentum. This opens a possibility to use the external spin bath as a stabilizer that alleviates destructive effects and restores the helicity protection.
\end{abstract}

DOI: 10.1103/PhysRevB.100.195426

\section{INTRODUCTION}

Topological insulators (TIs) are characterized by the existence of protected helical edge states-one-dimensional helical modes at the edges of two-dimensional TIs, and two-dimensional massless Dirac fermions at the surfaces of three-dimensional TIs [1-7]. This is a manifestation of a very general "bulk-edge correspondence" principle [8-11] which is probably one of the brightest applications of topological and geometrical concepts in condensed-matter physics. Importantly, topological protection of the edge states is not absolute: they can be broken by spin-dependent scattering mechanisms such as scattering on magnetic impurities $[6,12-$ 14] or electron-electron interactions [15]. These factors result in the backscattering and destruction of the helical modes, due to the intimate relation between their propagation direction and the direction of spins: if one flips the spin, one reverses the momentum. This effect has been considered from many perspectives, and a variety of its possible physical consequences on the transport and spectral properties of helical channels have been studied (see, e.g., [16,17]). For a detailed study of the effect of static magnetic impurities on the backscattering we refer the reader to ${ }^{1}[18,19]$. An interesting mechanism of formation of such magnetic moments from charge puddles has been suggested and analyzed in [20,21]. Similarly, emergence of magnetic scatterers from nonmagnetic impurities in combination with on-site electron-electron interactions was recently demonstrated in [22].

\footnotetext{
*a.bagrov@ science.ru.nl

†paco.guinea@gmail.com

$¥$ m.katsnelson@science.ru.nl

${ }^{1}$ It is particularly interesting to note that even the static impurities do not always lead to destruction of the quantized conductance, but only in the case of anisotropic exchange [19].
}

Because of the importance of practical implications of helical edge modes, such as ultrafast high-performance electronics or topological quantum computers [23], it is interesting to think of possible ways to reduce (or even eliminate) backscattering and make the edge modes more stable. An elegant approach to solving this problem can be deduced from the decoherence program in quantum physics $[24,25]$ (for the recent critical discussion of it, see [26]). According to this program, instant interactions between the system and the environment can be thought of as effective von Neumann projective measurements, and if the interactions are strong enough, they tend to stabilize the system in eigenstates of the interaction Hamiltonian. In the context of helical edge modes, it is tempting to think of a way to define projective measurements that stabilize states of well-defined momentum. Since momentum of a helical mode is coupled to its spin direction, one can try to attain the goal by making the spins classical via the "orthogonality catastrophe": if the environment degrees of freedom get entangled with spin-up and spin-down states of the system, the small overlap of the corresponding wave functions would suppress the amplitude of spin-flip processes [27-29].

Relying on this intuition, we suggest to couple the channel to a spin environment. While environment consisting of static spin degrees of freedom acts as a set of magnetic impurities that induce and amplify backscattering [30,31], the physics of fast itinerant spins can be very different, as known in the theory of magnetic resonance $[32,33]$. Indeed, in that case a strong static disorder broadens the line, due to a distribution of local resonant frequencies, whereas strong dynamic fluctuations narrow the line, due to averaging of the modulated fields (exchange or motional narrowing).

A related model, where dissipation induces decoherence in a Luttinger liquid, has been studied in [34]. The prototype model for the orthogonality suppression is a polaronic band narrowing [35,36]: if the on-site electron-phonon interaction energy is larger than the hopping amplitude (antiadiabatic 
regime) the latter is renormalized by an overlap function of phonon ground states at the electron situated at the left site and at the electron situated at the right site. In this sense, the localized on-site electron states are stabilized by the interaction with phonon environment: the probability amplitude to jump to another side is exponentially suppressed compared to the case of no electron-phonon interaction.

For the edge modes, this would mean stabilization of the states with definite momenta because the inversion of the propagation direction implies the spin reversal, but the probability amplitude of such a process is suppressed by the overlap of the corresponding ground states of the spin bath. In the terminology of Zurek [24], the states with a definite propagation direction appear to be "pointer states," robust upon the interactions with the environment.

Here we provide a formal analysis of the effect the environment has on the backscattering of helical states, using the renormalization-group approach similar to the one used in Refs. [28,37-40]. It turns out that, depending on the ratio of the exchange and direct interactions, the environment can both suppress and enhance the backscattering.

The paper is organized as follows. In the next section, we introduce the model of a helical channel interacting with environment. In Sec. III, we derive 1-loop renormalization group equations for this model. In Sec. IV, we solve these equations numerically for the case of $\mathrm{Bi}_{2} \mathrm{Te}_{3}$ topological insulator and construct phase diagram of the model. Finally, in Sec. V, we discuss possible implications of our results. In the Appendixes, we provide some additional results for another type of $1 \mathrm{~d}$ channel, InAs/GaSb quantum well, where the effect of suppression seems more pronounced, and discuss the possible role of spin-orbital coupling.

\section{MODEL}

We start with the following one-dimensional $s-d$ model, which, albeit simple, captures all the relevant aspects of more complicated and peculiar systems:

$$
\begin{aligned}
\mathcal{H}= & \sum_{k} c^{\dagger}(k) H^{c}(k) c(k)+\sum_{k ; i=1,2} d_{i}^{\dagger}(k) H_{i}^{d}(k) d_{i}(k) \\
& -J \sum_{q}\left(\sum_{k} c^{\dagger}(k) \vec{\sigma} c(k+q)\right) \\
& \times\left(\sum_{p ; i=1,2} d_{i}^{\dagger}(p) \vec{\sigma} d_{i}(p-q)\right)
\end{aligned}
$$

where $\vec{\sigma}$ are the Pauli matrices, $k$ is the one-dimensional spatial momentum, and the standard notation is used:

$$
\sum_{k}=\int_{-\pi / a}^{\pi / a} \frac{a d k}{2 \pi}
$$

where $a$ is the lattice constant. Here $c(k)$ and $d_{1,2}(k)$ are the helical edge modes of topological insulator and the environment degrees of freedom, respectively:

$$
c(k)=\left(c^{\uparrow}(k), c^{\downarrow}(k)\right), \quad d_{i}(k)=\left(d_{i}^{\uparrow}(k), d_{i}^{\downarrow}(k)\right),
$$

and the Hamiltonians of each sector are given by

$$
\begin{aligned}
& H^{c}(k)=\left(\begin{array}{cc}
\hbar v_{F} k & h_{0} \\
h_{0} & -\hbar v_{F} k
\end{array}\right), \\
& H_{1,2}^{d}(k)=\left(\begin{array}{cc} 
\pm \hbar c k & 0 \\
0 & \pm \hbar c k
\end{array}\right) .
\end{aligned}
$$

The bare backscattering is introduced via the off-diagonal term $h_{0}$ of the edge modes Hamiltonian. A concrete mechanism that induces backscattering is not important for our considerations. Since there is no preferred helicity in the environment, we take into account both right-moving ( $i=$ 1) and left-moving $(i=2)$ particles, and represent them for simplicity as two independent fermionic flavors. The chosen environment Hamiltonian looks like a perfect scattering-free channel, and we need to comment on how legitimate this assumption is. Of course, in a general case $H_{d}$ would have offdiagonal terms which might have some effect on the physics. However, in what follows, we will analyze how the parameter $h_{0}$ controlling backscattering in the edge channel changes due to the interactions with the spin environment, relying upon the perturbative renormalization-group approach [28,37-40]. Given that the off-diagonal terms of $H_{d}$ are not very large (let's denote them $h_{e}$ ), they will enter only the subleading terms of the perturbative expansion, $\sim h_{e} J J$, and would not alternate the leading-order renormalization-group (RG) flow equations. Therefore we impose $h_{e}=0$ from the very beginning, bearing in mind that our considerations remain valid for a "dirty" environment within the adopted level of approximation.

As will be evident, other interaction channels will be induced on top of the isotropic spin-spin interaction introduced in the Hamiltonian (1), and it turns out to be convenient to include them into the original Hamiltonian as a generalized vertex:

$$
\begin{gathered}
\mathcal{H}_{\text {int }}=\Gamma_{\alpha \beta \gamma \delta}^{(i)} \sum_{q, p, k} c_{\alpha}^{\dagger}(k) c_{\beta}(k+q) d_{i, \gamma}^{\dagger}(p) d_{i, \delta}(p-q), \\
\Gamma_{\alpha \beta \gamma \delta}^{(i)}=J_{00}^{(i)} \mathbb{I}_{\alpha \beta} \otimes \mathbb{I}_{\gamma \delta}+J_{z z}^{(i)} \sigma_{\alpha \beta}^{z} \otimes \sigma_{\gamma \delta}^{z} \\
+J^{(i)}\left(\sigma_{\alpha \beta}^{x} \otimes \sigma_{\gamma \delta}^{x}+\sigma_{\alpha \beta}^{y} \otimes \sigma_{\gamma \delta}^{y}\right) \\
+J_{0 z}^{(i)} \mathbb{I}_{\alpha \beta} \otimes \sigma_{\gamma \delta}^{z}+J_{z 0}^{(i)} \sigma_{\alpha \beta}^{z} \otimes \mathbb{I}_{\gamma \delta},
\end{gathered}
$$

where we also added the Coulomb channel $J_{00}$, the spincharge channels $J_{0 z}$ and $J_{z 0}$, and the possible anisotropy between $Z$ and $X Y$ spin couplings. This reduces to the isotropic spin interaction of (1) if

$$
J_{00}^{(i)}=J_{0 z}^{(i)}=J_{z 0}^{(i)}=0, \quad J_{z z}^{(i)}=J^{(i)}=J .
$$

To make the notations more handy and reduce the number of indices, hereinafter we denote the coupling constants $J^{(1)}$ as plain $J$, and $J^{(2)}$ as $\tilde{J}$.

\section{RENORMALIZATION-GROUP FLOW EQUATIONS}

As we elaborated on in the Introduction, we expect the spin-spin interactions between the edge of the topological insulator and the bath to make pointer states of the system to be states with well-defined spin, and thus stabilize the helical modes. In terms of the renormalization-group flow for the model (6), (7), it means that the mode-mixing parameter $h$ is expected to become irrelevant in the infrared. 


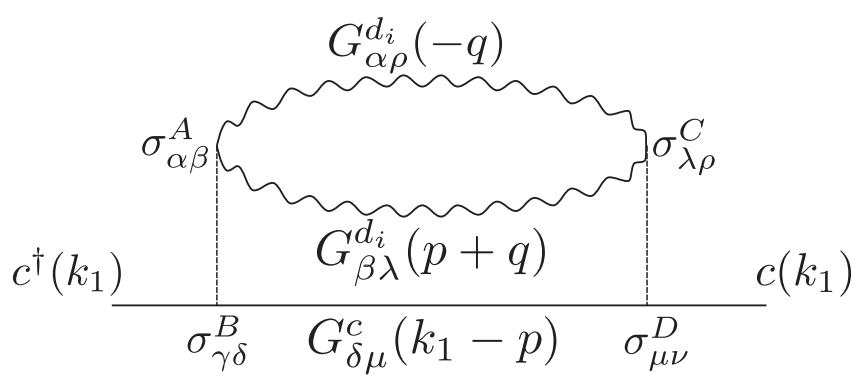

FIG. 1. Self-energy correction to the helical edge modes. Wavy lines denote the propagators of the environment modes. Latin letters stand for $x, y, z$, and the Greek ones denote the spin indices. Sum over all combinations of $A, B, C, D$ allowed by the structure of vertex (7) has to be taken.

The leading-order quantum correction to $h$ is given by the off-diagonal part of the two-loop self-energy diagram shown in Fig. 1 (from now on all calculations will be conducted for the Matsubara Green's functions):

$$
\begin{aligned}
G_{c}^{-1}(i \omega, k) & =G_{c, 0}^{-1}(i \omega, k)-\Sigma(i \omega, k), \\
h(i \omega, k) & =h_{0}+\Sigma_{01}(i \omega, k),
\end{aligned}
$$

where the bare Green's function of edge fermions is related to their Hamiltonian (4) as

$$
G_{c, 0}^{-1}(i \omega, k)=i \omega \mathbb{I}-H^{c}(k)
$$

The polarization loop is given by a simple integral:

$$
\begin{aligned}
\Pi_{1,2}^{A C}(p)= & \int_{-\pi / a}^{\pi / a} \frac{a d q}{2 \pi} \int_{-\infty}^{\infty} \frac{d \omega_{q}}{2 \pi} \operatorname{Tr}\left[\sigma^{A} G^{d_{1,2}}\left(i \omega_{p}+i \omega_{q}, p+q\right)\right. \\
& \left.\times \sigma^{C} G^{d_{1,2}}\left(-i \omega_{q},-q\right)\right] \\
= & \frac{a p}{\pi\left(\mp i \omega_{p}+\hbar c p\right)} \delta^{A C} .
\end{aligned}
$$

To obtain the self-energy correction, we need to sum over all possible combinations of $A, B, C, D$ indices in Fig. 1 that give nontrivial contributions, as well as over the two flavors of the environment modes. The resulting expression at zero external momentum is

$$
\begin{aligned}
\Sigma_{01}(0,0)= & -\int_{-\pi / a}^{\pi / a} \frac{a d q}{2 \pi} \int_{-\infty}^{\infty} \frac{d \omega_{p}}{2 \pi} \frac{a h q}{\left(h^{2}+\omega_{q}^{2}+\hbar^{2} v_{F}^{2} q^{2}\right)} \\
& \times\left(\frac{\alpha(J)}{i \pi \omega_{q}-\pi \hbar c q}-\frac{\alpha(\tilde{J})}{i \pi \omega_{q}+\pi \hbar c q}\right),
\end{aligned}
$$

where we introduced

$$
\alpha(J)=J_{00}^{2}+J_{0 z}^{2}-J_{z 0}^{2}-J_{z z}^{2} .
$$

Although there is a natural ultraviolet (UV) cutoff given by the lattice constant $a$, it is convenient to formally consider the momentum integral over the second loop as logarithmically divergent in the $a \rightarrow 0$ limit, as it allows to extract the leading scaling that defines the renormalizationgroup flow. Evaluating the integral over frequencies via residues, and then expanding the integrand around $|q| \rightarrow \infty$, we obtain the correction to backscattering amplitude from a thin momentum shell $|q| \in$ $[\Lambda, \Lambda+d \Lambda]$ :

$$
\begin{aligned}
h(\Lambda+d \Lambda) & =h(\Lambda)+\delta \Sigma_{01}=h(\Lambda)-\frac{2}{4 \pi^{2}} \int_{\Lambda}^{\Lambda+d \Lambda} \frac{a^{2} h(q)[\alpha(J)+\alpha(\tilde{J})] d q}{\sqrt{h(q)^{2}+\hbar^{2} v_{F}^{2} q^{2}}\left(\hbar c q+\sqrt{h(q)^{2}+\hbar^{2} v_{F}^{2} q^{2}}\right)} \\
& =h(\Lambda)-\frac{1}{2 \pi^{2}} h(\Lambda) \int_{\Lambda}^{\Lambda+d \Lambda} \frac{a^{2}[\alpha(J)+\alpha(\tilde{J})] d q}{\hbar^{2} v_{F}\left(c+v_{F}\right) q},
\end{aligned}
$$

where the additional overall factor of 2 is due to integration over both positive and negative momenta. That is, we obtain the corresponding flow equation:

$$
\frac{d h}{d \ln \Lambda}=-\frac{a^{2} h}{2 \pi^{2} \hbar^{2} v_{F}\left(c+v_{F}\right)}[\alpha(J)+\alpha(\tilde{J})] .
$$

If we ignore for a moment renormalization of other parameters of the model, we can readily conclude that

$$
h(\Lambda)=h_{0}\left(\frac{\Lambda}{\Lambda_{\mathrm{UV}}}\right)^{\gamma}
$$

where for further convenience we introduce a notation for the exponent, as it serves as a good measure of the "irrelevance" of backscattering:

$$
\gamma=-\frac{a^{2}}{2 \pi^{2} \hbar^{2} v_{F}\left(c+v_{F}\right)}[\alpha(J)+\alpha(\tilde{J})] .
$$

If only spin-spin interactions are present

$$
\alpha(J)+\alpha(\tilde{J})=-J_{z z}^{2}-\tilde{J}_{z z}^{2},
$$

and the mode mixing is clearly irrelevant in the infrared limit $\Lambda \rightarrow 0(\gamma>0)$.

However, this naive treatment is incomplete; to obtain a full picture of interaction effects in this model also requires taking into account renormalization of the coupling matrices $\Gamma^{(1,2)}$, and the Fermi velocities $v_{F}$ and $c$.

Renormalization of the couplings is given by the one-loop vertex diagram shown in Fig. 2. The corresponding momentum integral is also logarithmically divergent, and, omitting the intermediate steps similar to what we have done when computing the backscattering amplitude renormalization, we arrive at the following system of RG flow equations:

$$
\begin{aligned}
\frac{d J}{d \ln \Lambda} & =\frac{a}{\pi \hbar\left(c+v_{F}\right)} J\left(J_{00}+J_{0 z}-J_{z 0}-J_{z z}\right) \\
\frac{d \tilde{J}}{d \ln \Lambda} & =\frac{a}{\pi \hbar\left(c+v_{F}\right)} \tilde{J}\left(\tilde{J}_{00}-\tilde{J}_{0 z}+\tilde{J}_{z 0}-\tilde{J}_{z z}\right),
\end{aligned}
$$




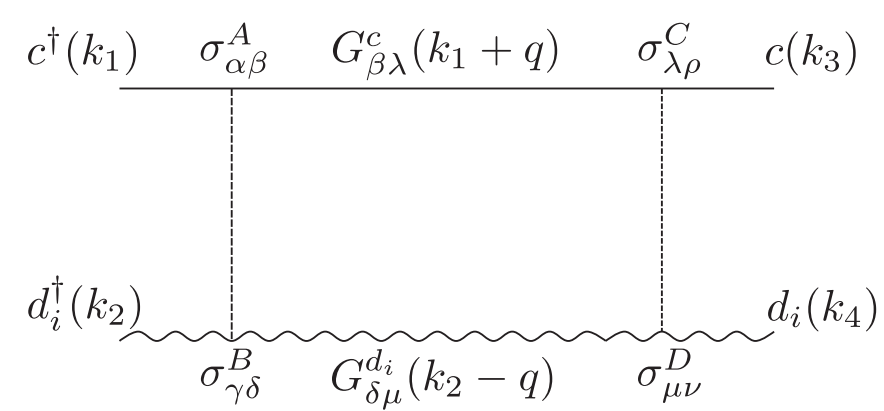

FIG. 2. Vertex correction to the coupling matrices $\Gamma^{(1,2)}$.

$$
\begin{aligned}
\frac{d J_{00}}{d \ln \Lambda} & =\frac{a}{2 \pi \hbar\left(c+v_{F}\right)}\left[2 J^{2}+\left(J_{00}-J_{z 0}\right)^{2}+\left(J_{0 z}-J_{z z}\right)^{2}\right] \\
\frac{d \tilde{J}_{00}}{d \ln \Lambda} & =\frac{a}{2 \pi \hbar\left(c+v_{F}\right)}\left(2 \tilde{J}^{2}+\left(\tilde{J}_{00}+\tilde{J}_{z 0}\right)^{2}+\left(\tilde{J}_{0 z}+\tilde{J}_{z z}\right)^{2}\right), \\
\frac{d J_{0 z}}{d \ln \Lambda} & =-\frac{a}{\pi \hbar\left(c+v_{F}\right)}\left[J^{2}-\left(J_{00}-J_{z 0}\right)\left(J_{0 z}-J_{z z}\right)\right] \\
\frac{d \tilde{J}_{0 z}}{d \ln \Lambda} & =\frac{a}{\pi \hbar\left(c+v_{F}\right)}\left(\tilde{J}^{2}+\left(\tilde{J}_{00}+\tilde{J}_{z 0}\right)\left(\tilde{J}_{0 z}+\tilde{J}_{z z}\right)\right), \\
\frac{d J_{z 0}}{d \ln \Lambda} & =\frac{a}{2 \pi \hbar\left(c+v_{F}\right)}\left[2 J^{2}-\left(J_{00}-J_{z 0}\right)^{2}-\left(J_{0 z}-J_{z z}\right)^{2}\right] \\
\frac{d \tilde{J}_{z 0}}{d \ln \Lambda} & =\frac{a}{2 \pi \hbar\left(c+v_{F}\right)}\left(-2 \tilde{J}^{2}+\left(\tilde{J}_{00}+\tilde{J}_{z 0}\right)^{2}+\left(\tilde{J}_{0 z}+\tilde{J}_{z z}\right)^{2}\right), \\
\frac{d J_{z z}}{d \ln \Lambda} & =-\frac{a}{\pi \hbar\left(c+v_{F}\right)}\left[J^{2}+\left(J_{00}-J_{z 0}\right)\left(J_{0 z}-J_{z z}\right)\right] \\
\frac{d \tilde{J}_{z z}}{d \ln \Lambda} & =\frac{a}{\pi \hbar\left(c+v_{F}\right)}\left(-\tilde{J}^{2}+\left(\tilde{J}_{00}+\tilde{J}_{z 0}\right)\left(\tilde{J}_{0 z}+\tilde{J}_{z z}\right)\right) .
\end{aligned}
$$

Fermi velocity renormalization comes from the diagonal part of the self-energy diagram, Fig. 1. Formally speaking, there are two different Fermi velocities for the two edge helical modes that renormalize independently:

$$
\begin{aligned}
& \frac{d v_{F}}{d \ln \Lambda}=-\frac{4 a^{2}}{\pi^{2} \hbar^{2}\left(c+v_{F}\right)^{2}} v_{F} J^{2}, \\
& \frac{d \tilde{v}_{F}}{d \ln \Lambda}=-\frac{4 a^{2}}{\pi^{2} \hbar^{2}\left(c+\tilde{v}_{F}\right)^{2}} \tilde{v}_{F} \tilde{J}^{2},
\end{aligned}
$$

but we can consistently assume symmetry between them, and impose $J=\tilde{J}, v_{F}=\tilde{v}_{F}$ at all scales.

In principle, we also have to derive the renormalizationgroup flow for the Fermi velocity $c$, but since $v_{F} \gg c$ in the cases of interest (when the discussed renormalization of backscattering amplitude is strong), and they appear in $1 /\left(v_{F}+c\right)$ combination, renormalization of the bath Fermi velocity can be neglected.

If symmetry between the bare couplings is imposed, $J_{00}=$ $\tilde{J}_{00}, J_{z z}=\tilde{J}_{z z}$, the flow equations assume the simplified form:

$$
\begin{aligned}
\frac{d J}{d \ln \Lambda} & =\frac{a}{\pi \hbar\left(c+v_{F}\right)} J\left(J_{00}+J_{0 z}-J_{z 0}-J_{z z}\right), \\
\frac{d J_{00}}{d \ln \Lambda} & =\frac{a}{2 \pi \hbar\left(c+v_{F}\right)}\left[2 J^{2}+\left(J_{00}-J_{z 0}\right)^{2}+\left(J_{0 z}-J_{z z}\right)^{2}\right],
\end{aligned}
$$

$$
\begin{aligned}
\frac{d J_{0 z}}{d \ln \Lambda} & =-\frac{a}{\pi \hbar\left(c+v_{F}\right)}\left[J^{2}-\left(J_{00}-J_{z 0}\right)\left(J_{0 z}-J_{z z}\right)\right] \\
\frac{d J_{z 0}}{d \ln \Lambda} & =\frac{a}{2 \pi \hbar\left(c+v_{F}\right)}\left[2 J^{2}-\left(J_{00}-J_{z 0}\right)^{2}-\left(J_{0 z}-J_{z z}\right)^{2}\right] \\
\frac{d J_{z z}}{d \ln \Lambda} & =-\frac{a}{\pi \hbar\left(c+v_{F}\right)}\left[J^{2}+\left(J_{00}-J_{z 0}\right)\left(J_{0 z}-J_{z z}\right)\right] \\
\frac{d v_{F}}{d \ln \Lambda} & =-\frac{4 a^{2}}{\pi^{2} \hbar^{2}\left(c+v_{F}\right)^{2}} v_{F} J^{2}, \\
\frac{d h}{d \ln \Lambda} & =-\frac{a^{2} h}{\pi^{2} \hbar^{2} v_{F}\left(c+v_{F}\right)}\left(J_{00}^{2}+J_{0 z}^{2}-J_{z 0}^{2}-J_{z z}^{2}\right) \\
\tilde{J} & =J, \quad \tilde{J}_{00}=J_{00}, \quad \tilde{J}_{z z}=J_{z z}, \quad \tilde{J}_{z 0}=-J_{z 0}, \\
\tilde{J}_{0 z} & =-J_{0 z} .
\end{aligned}
$$

We should keep in mind that it is also possible to have spin-orbital couplings like $J_{0 x}, J_{x 0}, J_{y 0}, J_{0 y}$, but as we show in Appendix A, they do not play any significant role as long as they are less than $\sim 0.3 J, J_{00}$.

\section{PHASE DIAGRAM OF THE MODEL}

Solving flow equations (21) numerically in different regimes, we can identify how the backscattering of helical modes is affected by the environment. To make numerical estimates, we need to agree on the values of bare physical quantities. Fermi velocity of the edge degrees of freedom in two-dimensional $\mathrm{Bi}_{2} \mathrm{Te}_{3}$ topological insulators is measured to be $v_{F} \simeq 5 \times 10^{7} \mathrm{~cm} / \mathrm{s}$ [41]. The spin bath velocity $c$ is a free parameter that can be tuned to any value by choosing a proper environment material, and we find the effect of backscattering suppression to be stronger when $c$ is small, $\sim 10^{7} \mathrm{~cm} / \mathrm{s}$, i.e., when the bath is insulating. The in-plane lattice constant for $\mathrm{Bi}_{2} \mathrm{Te}_{3}$ is $a=6.67 \AA$. It is interesting to study the model in different regimes and analyze both the role of the spin-spin and the Coulomb interactions, and their interplay.

Thus, we will take the bare backscattering amplitude $h=$ $0.1 \mathrm{eV}$, and focus on three different cases:

(i) The Coulomb interaction is dominant:

$$
\begin{gathered}
J_{00}=\tilde{J}_{00}=0.2 \mathrm{eV}, \\
J=\tilde{J}=J_{z z}=\tilde{J}_{z z}=0 .
\end{gathered}
$$

The energy gap in $\mathrm{Bi}_{2} \mathrm{Te}_{3}$ is $\Delta E \simeq 0.34 \mathrm{eV}$, so we do not want the exchange interactions to be larger than that.

(ii) The spin-spin channel is dominant:

$$
\begin{gathered}
J=\tilde{J}=J_{z z}=\tilde{J}_{z z}=0.2 \mathrm{eV}, \\
J_{00}=\tilde{J}_{00}=0 .
\end{gathered}
$$

While this case seems quite special since normally the Coulomb interactions are stronger than the $s$ - $d$ exchange, it is instructive to consider this regime as it shows a possibility to use the environment to suppress backscattering and enhance protection of the helical edge modes.

(iii) Spin and Coulomb interactions are comparable:

$$
J=\tilde{J}=J_{z z}=\tilde{J}_{z z}=J_{00}=\tilde{J}_{00}=0.2 \mathrm{eV} .
$$




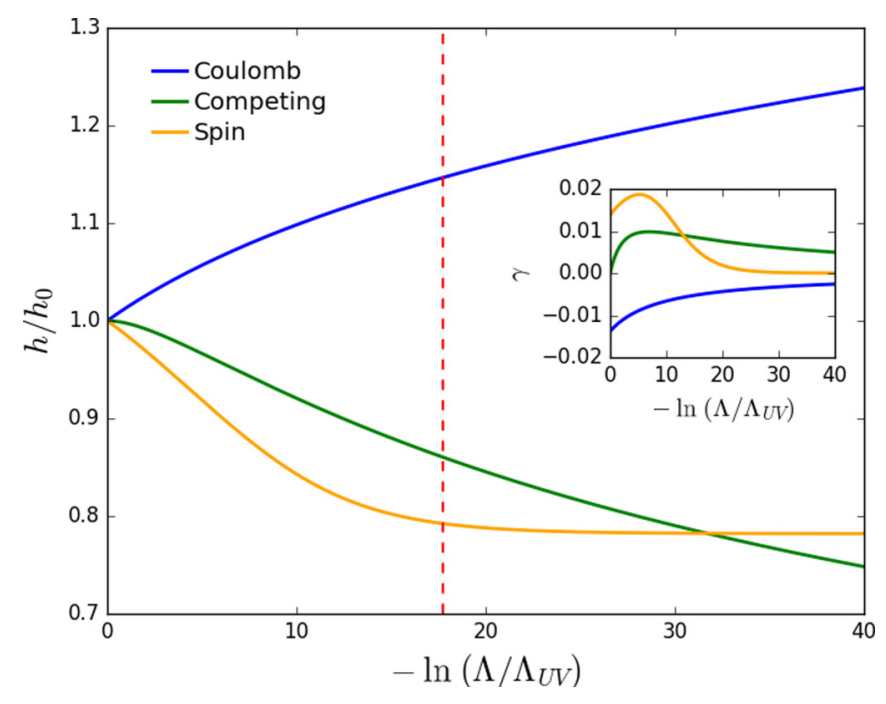

FIG. 3. RG flows of the backscattering amplitude. The blue curve depicts the Coulomb-interaction dominated case $\left(J_{00}=\right.$ $0.2 \mathrm{eV}, J_{z z}=0 \mathrm{eV}$ ), the yellow one depicts the case of dominant spin-spin interaction channel $\left(J_{00}=0 \mathrm{eV}, J_{z z}=0.2 \mathrm{eV}\right)$, and the green one depicts the regime of interplay $\left(J_{00}=0.2 \mathrm{eV}, J_{z z}=\right.$ $0.2 \mathrm{eV}$ ). The dashed line depicts the energy scale corresponding to temperature about $T=20 \mathrm{~K}$. Inset: the corresponding flows of the "irrelevance" parameter $\gamma$. The UV cutoff is taken to be $\Lambda_{\mathrm{UV}}=10 a$, which is an estimate for the penetration depth of the edge modes into the bulk $[18,42]$.

This case appears to be the most nontrivial one as we will see below.

The numerical solution to the systems of the renormalization-group equations in the three mentioned regimes is shown in Fig. 3. One can see that while the pure Coulomb interaction causes enhancement of backscattering, its interplay with the spin-spin coupling and the other induced interaction channels is highly nontrivial. At intermediate energies, if the two competing channels are present, Coulomb reduces the effect of suppressing. However, if one goes to lower energies, it assists the spin interactions in suppressing the process of backscattering, and makes $h$ flowing to zero even when capacity of the spin channel is exhausted, and renormalization of $h$ stopped.

Another way to see this is to define again the "irrelevance parameter" $\gamma(\Lambda)$ (now it is scale dependent) by formally representing the solution to the equation on $h$ as

$$
h(\Lambda)=h_{0}\left(\frac{\Lambda}{\Lambda_{\mathrm{UV}}}\right)^{\gamma(\Lambda)} .
$$

Dynamics of $\gamma$ is shown in the inset of Fig. 3. Though Coulomb interaction decreases the initial value of $\gamma$, deep in the infrared it prevents $\gamma$ from flowing to zero. The difference between the two regimes looks rather mild, but since the coupling constants flow towards strong coupling in the infrared, the leading-order perturbative analysis tends to underestimate renormalization of $\gamma$, and a stronger effect can be expected.

The full phase diagram scanned over different values of $J_{00}$ and $J=J_{z z}$ is shown in Fig. 4. One can see that all the three cases are quite generic, and occur within large domains of the parametric space. It also can be seen that in the formal limit

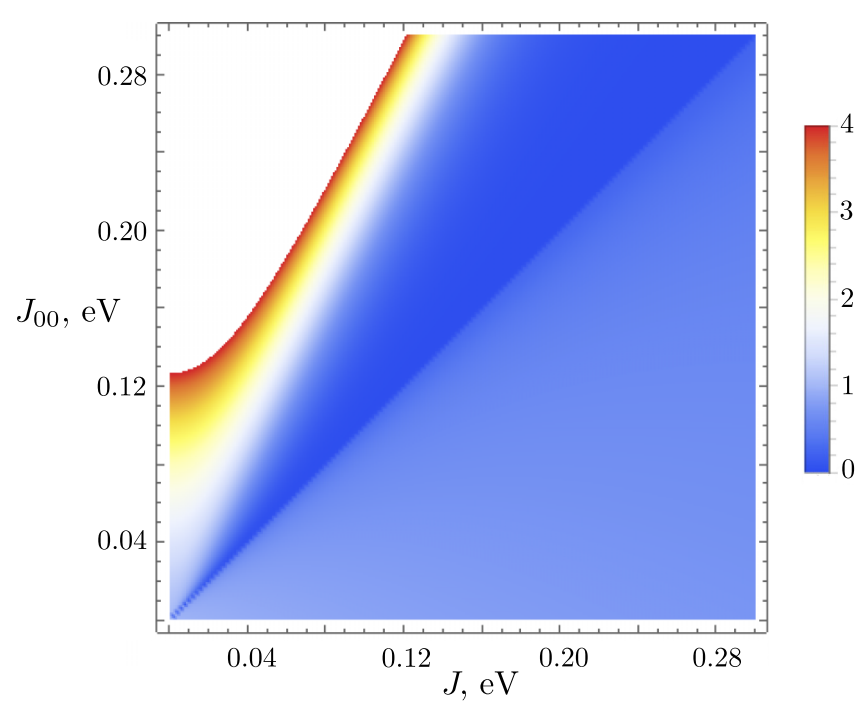

FIG. 4. Density plot of the renormalized backscattering amplitude $h / h_{0}$ as a function of bare spin-spin $(J)$ and Coulomb $\left(J_{00}\right)$ interactions. The three discussed regimes are clearly seen here. In particular, for the dark blue wedge in the middle, numerical extrapolation of solutions to Eq. (21) shows that $h(\Lambda) / h_{0} \rightarrow 0$ as $\Lambda \rightarrow 0$.

$\Lambda \rightarrow 0$, the backscattering amplitude $h$ is taken to zero in the case of competing interactions.

In all these cases we take the bare couplings $J_{0 z}, J_{z 0}, \tilde{J}_{0 z}, \tilde{J}_{z 0}$ equal to zero. Since they flow nontrivially upon renormalization, it is worth discussing how they could affect the outlined physical picture. First of all, while it is natural to assume that such spin-orbital couplings are much smaller than the spin-spin and the Coulomb interactions, what really matters here is the asymmetry $J_{0 z}^{2}-J_{z 0}^{2}$. Even if these constants are pretty large, $\sim 0.5 J_{z z}$, but the symmetry $J_{0 z}=J_{z 0}$ is retained, the phase diagram in Fig. 4 remains unchanged. However, large asymmetry between them can destroy the "irrelevant" phase.

\section{DISCUSSION AND CONCLUSIONS}

In this paper, taking the intuition provided by the concept of pointer states as an inspiration, we have studied how interactions with environment affect the backscattering of helical modes in edge channels by deriving the leading-order perturbative renormalization-group flow equations. We have discovered that the interplay of the Coulomb and spin-spin interactions between the modes and the environment leads to a nontrivial phase diagram. Dominance of the Coulomb interaction expectedly leads to amplification of the backscattering, making helicity of the propagating modes poorly defined. If only the spin-spin interaction channel is present, the backscattering gets marginally suppressed along the RG flow, receiving a finite negative correction to its bare amplitude. This is in line with what can be qualitatively expected from the basic consideration in terms of decoherence and pointer states, as discussed in the Introduction. However, our quantitative analysis gives much more. The most interesting situation turns out to be when both the interaction channels are at 
work. Then the Coulomb interaction assists the spin-spin one in suppressing backscattering, making backscattering rather irrelevant than marginally suppressed. The conducted analysis allows us to conclude that the external bath of itinerant spins can be not only dangerous for the helicity of modes in the channel, but also, in certain regimes, can serve as a stabilizer and alleviate the destructive effect of backscattering, restoring the protection of the helical modes. For a particular example of archetypical 2D topological insulator $\mathrm{Bi}_{2} \mathrm{Te}_{3}$, we have estimated that interactions with environment can reduce the backscattering amplitude to $\sim 75 \%$ of its original value within a physically reasonable range of energy scales. In systems with smaller Fermi velocities, like InAs/GaSb quantum wells studied among other structures in [30,31] $\left(v_{F}=4.6 \times 10^{6} \mathrm{~cm} / \mathrm{s}, a=6.1 \AA\right)$, the suppression is even more pronounced, and the backscattering amplitude will be reduced to $20 \%$ of its original value or even stronger (see Appendix B). One should also keep in mind that the leadingorder perturbative expansion might provide only the lower bound on the strength of the effect, as the deep IR limit of the model is strongly coupled, and a stronger suppression is expected in the nonperturbative domain, making the studied mechanism a good candidate for protecting helical modes.

\section{ACKNOWLEDGMENTS}

We are thankful to E. Stepanov for useful discussions. The work was supported by ERC Advanced Grant No. 338957 FEMTO/NANO and by the NWO Spinoza Prize of Mikhail Katsnelson (2013).

\section{APPENDIX A: THE CASE OF InAs/GaSb QUANTUM WELLS}

While the effect of suppression of the backscattering on the edge of topological insulators is notable but not ultimate leading to decrease in the backscattering amplitude $h$ to $\sim 80 \%$ of its original value, it is natural to pose a question of whether there are systems where it is more pronounced. Since the effective dimensionless coupling constant is defined as $J a /\left(2 \pi \hbar v_{F}\right)$, we can expect stronger suppression in materials with smaller Fermi velocities. One possibility is InAs/GaSb quantum wells studied in [30,31]. Then $v_{F}=$ $4.6 \times 10^{6} \mathrm{~cm} / \mathrm{s}, c=10^{6} \mathrm{~cm} / \mathrm{s}, a=6.1 \AA$. Taking $J=0.3 \mathrm{eV}$ and $J_{00}=0$ or $0.3 \mathrm{eV}$, which corresponds to the limit of applicability of perturbative approach, we obtain the flow of $h$ shown in Fig. 5. Due to the smaller Fermi velocity in the channel, the suppression effect becomes much more significant, and $h$ renormalizes to $\sim 25-30 \%$ within a physically reasonable range of scales.

\section{APPENDIX B: INTERPLAY WITH THE SPIN-ORBIT COUPLING}

So far we have analyzed the minimally consistent set of couplings given by

$$
\begin{aligned}
\mathcal{H}_{\text {int }} & =\Gamma_{\alpha \beta \gamma \delta}^{(i)} \sum_{q, p, k} c_{\alpha}^{\dagger}(k) c_{\beta}(k+q) d_{i, \gamma}^{\dagger}(p) d_{i, \delta}(p-q), \\
\Gamma_{\alpha \beta \gamma \delta}^{(i)} & =J_{00}^{(i)} \mathbb{I}_{\alpha \beta} \otimes \mathbb{I}_{\gamma \delta}+J_{z z}^{(i)} \sigma_{\alpha \beta}^{z} \otimes \sigma_{\gamma \delta}^{z}
\end{aligned}
$$

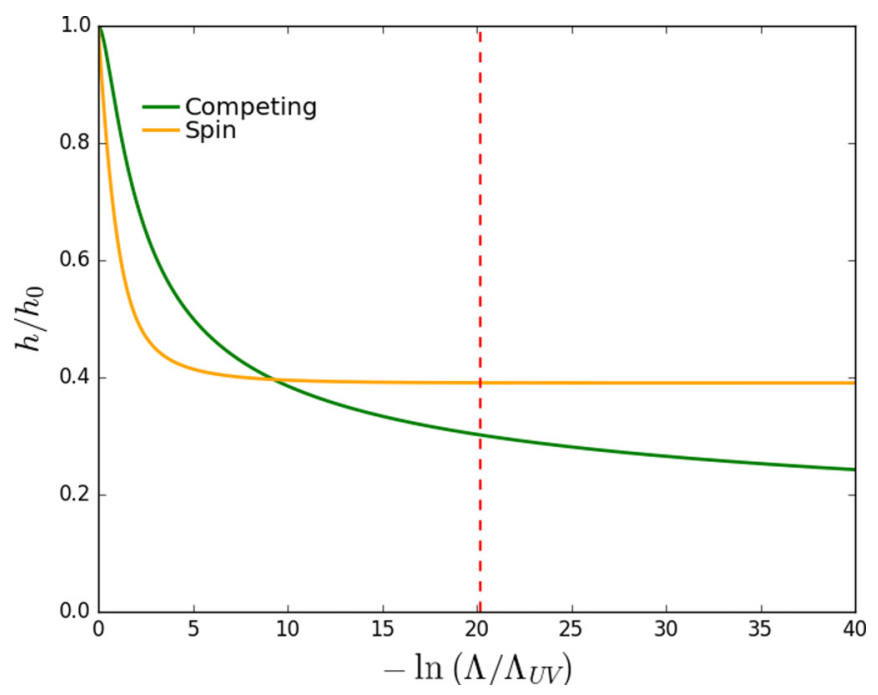

FIG. 5. Renormalization of the backscattering amplitude in InAs/GaSb quantum wells.

$$
\begin{aligned}
& +J^{(i)}\left(\sigma_{\alpha \beta}^{x} \otimes \sigma_{\gamma \delta}^{x}+\sigma_{\alpha \beta}^{y} \otimes \sigma_{\gamma \delta}^{y}\right)+J_{0 z}^{(i)} \mathbb{I}_{\alpha \beta} \otimes \sigma_{\gamma \delta}^{z} \\
& +J_{z 0}^{(i)} \sigma_{\alpha \beta}^{z} \otimes \mathbb{I}_{\gamma \delta},
\end{aligned}
$$

where $i=1,2$ are two channels in the bath. However, in a real system a spin-orbit coupling that tends to enhance backscattering can be present, and we have to make sure that the structure of the renormalization-group flow does not change drastically upon switching on such an interaction. To check that, we shall consider a more general form of coupling matrix and enlarge it by adding the following terms:

$$
\begin{aligned}
\Gamma_{\alpha \beta \gamma \delta}^{(i) \mathrm{SO}}= & J_{0 x}^{(i)} \mathbb{I}_{\alpha \beta} \otimes \sigma_{\gamma \delta}^{x}+J_{x 0}^{(i)} \sigma_{\alpha \beta}^{x} \otimes \mathbb{I}_{\gamma \delta}+J_{z x}^{(i)} \sigma_{\alpha \beta}^{z} \otimes \sigma_{\gamma \delta}^{x} \\
& +J_{x z}^{(i)} \sigma_{\alpha \beta}^{x} \otimes \sigma_{\gamma \delta}^{z} .
\end{aligned}
$$

The resulting system of one-loop RG flow equations reads

$$
\begin{aligned}
\frac{d J_{x x}}{d \ln \Lambda}= & \frac{a}{\pi \hbar\left(c+v_{F}\right)}\left(J_{0 x} J_{x 0}+J_{00} J_{x x}+J_{0 z} J_{y y}-J_{x x} J_{z 0}\right. \\
& \left.-J_{x 0} J_{z x}-J_{y y} J_{z z}\right), \\
\frac{d J_{y y}}{d \ln \Lambda}= & \frac{a}{\pi \hbar\left(c+v_{F}\right)}\left(J_{0 z} J_{x x}-J_{0 x} J_{x z}+J_{00} J_{y y}-J_{y y} J_{z 0}\right. \\
& \left.+J_{x z} J_{z x}-J_{x x} J_{z z}\right), \\
\frac{d J_{00}}{d \ln \Lambda}= & \frac{a}{2 \pi \hbar\left(c+v_{F}\right)}\left[J_{x 0}^{2}+J_{x x}^{2}+J_{y y}^{2}+J_{x z}^{2}+\left(J_{0 x}-J_{z x}\right)^{2}\right. \\
& \left.+\left(J_{00}-J_{z 0}\right)^{2}+\left(J_{0 z}-J_{z z}\right)^{2}\right], \\
\frac{d J_{0 z}}{d \ln \Lambda}= & \frac{a}{\pi \hbar\left(c+v_{F}\right)}\left[J_{x x} J_{y y}-J_{x 0} J_{x z}+\left(J_{00}-J_{z 0}\right)\left(J_{0 z}-J_{z z}\right)\right], \\
\frac{d J_{z 0}}{d \ln \Lambda}= & \frac{a}{2 \pi \hbar\left(c+v_{F}\right)}\left[J_{x 0}^{2}+J_{x x}^{2}+J_{y y}^{2}+J_{x z}^{2}-\left(J_{00}-J_{z 0}\right)^{2}\right. \\
& \left.-\left(J_{0 z}-J_{z z}\right)^{2}-\left(J_{0 x}-J_{z x}\right)^{2}\right], \\
\frac{d J_{z z}}{d \ln \Lambda}= & \frac{a}{\pi \hbar\left(c+v_{F}\right)}\left[-J_{x x} J_{y y}+J_{x 0} J_{x z}-\left(J_{00}-J_{z 0}\right)\right. \\
& \left.\times\left(J_{0 z}-J_{z z}\right)\right],
\end{aligned}
$$




$$
\begin{aligned}
& \frac{d J_{0 x}}{d \ln \Lambda}=\frac{a}{\pi \hbar\left(c+v_{F}\right)}\left[J_{x 0} J_{x x}+J_{x z} J_{y y}+\left(J_{00}-J_{z 0}\right)\right. \\
& \left.\times\left(J_{0 x}-J_{z x}\right)\right] \text {, } \\
& \frac{d J_{x 0}}{d \ln \Lambda}=\frac{a}{\pi \hbar\left(c+v_{F}\right)}\left(J_{00} J_{x 0}+J_{0 x} J_{x x}+J_{0 z} J_{x z}-J_{x 0} J_{z 0}\right. \\
& \left.-J_{x x} J_{z x}-J_{x z} J_{z z}\right) \text {, } \\
& \frac{d J_{x z}}{d \ln \Lambda}=\frac{a}{\pi \hbar\left(c+v_{F}\right)}\left(J_{0 z} J_{x 0}+J_{00} J_{x z}-J_{0 x} J_{y y}-J_{x z} J_{z 0}\right. \\
& \left.+J_{y y} J_{z x}-J_{x 0} J_{z z}\right) \text {, } \\
& \frac{d J_{z x}}{d \ln \Lambda}=\frac{a}{\pi \hbar\left(c+v_{F}\right)}\left[J_{x 0} J_{x x}+J_{x z} J_{y y}-\left(J_{00}-J_{z 0}\right)\right. \\
& \left.\times\left(J_{0 x}-J_{z x}\right)\right], \\
& \frac{d \tilde{J}_{x x}}{d \ln \Lambda}=\frac{a}{\pi \hbar\left(c+v_{F}\right)}\left(\tilde{J}_{x x} \tilde{J}_{00}+\tilde{J}_{x x} \tilde{J}_{z 0}+\tilde{J}_{x 0} \tilde{J}_{0 x}+\tilde{J}_{x 0} \tilde{J}_{z x}\right. \\
& \left.-\tilde{J}_{y y} \tilde{J}_{0 z}-\tilde{J}_{y y} \tilde{J}_{z z}\right) \text {, } \\
& \frac{d \tilde{J}_{y y}}{d \ln \Lambda}=\frac{a}{\pi \hbar\left(c+v_{F}\right)}\left(\tilde{J}_{y y} \tilde{J}_{00}+\tilde{J}_{y y} \tilde{J}_{z 0}+\tilde{J}_{x z} \tilde{J}_{0 x}+\tilde{J}_{x z} \tilde{J}_{z x}\right. \\
& \left.-\tilde{J}_{x x} \tilde{J}_{0 z}-\tilde{J}_{x x} \tilde{J}_{z z}\right) \text {, } \\
& \frac{d \tilde{J}_{00}}{d \ln \Lambda}=\frac{a}{2 \pi \hbar\left(c+v_{F}\right)}\left(\tilde{J}_{x 0}^{2}+\tilde{J}_{x x}^{2}+\tilde{J}_{y y}^{2}+\tilde{J}_{x z}^{2}+\left(\tilde{J}_{0 x}+\tilde{J}_{z x}\right)^{2}\right. \\
& \left.+\left(\tilde{J}_{00}+\tilde{J}_{z 0}\right)^{2}+\left(\tilde{J}_{0 z}+\tilde{J}_{z z}\right)^{2}\right) \text {, } \\
& \frac{d \tilde{J}_{0 z}}{d \ln \Lambda}=\frac{a}{\pi \hbar\left(c+v_{F}\right)}\left(\tilde{J}_{x x} \tilde{J}_{y y}+\tilde{J}_{x 0} \tilde{J}_{x z}+\left(\tilde{J}_{00}+\tilde{J}_{z 0}\right)\right. \\
& \left.\times\left(\tilde{J}_{0 z}+\tilde{J}_{z z}\right)\right), \\
& \frac{d \tilde{J}_{z 0}}{d \ln \Lambda}=\frac{a}{2 \pi \hbar\left(c+v_{F}\right)}\left(-\tilde{J}_{x 0}^{2}-\tilde{J}_{x x}^{2}-\tilde{J}_{y y}^{2}-\tilde{J}_{x z}^{2}\right. \\
& \left.+\left(\tilde{J}_{00}+\tilde{J}_{z 0}\right)^{2}+\left(\tilde{J}_{0 z}+\tilde{J}_{z z}\right)^{2}+\left(\tilde{J}_{0 x}+\tilde{J}_{z x}\right)^{2}\right), \\
& \frac{d \tilde{J}_{z z}}{d \ln \Lambda}=\frac{a}{\pi \hbar\left(c+v_{F}\right)}\left(-\tilde{J}_{x x} \tilde{J}_{y y}-\tilde{J}_{x 0} \tilde{J}_{x z}+\left(\tilde{J}_{00}+\tilde{J}_{z 0}\right)\right. \\
& \left.\times\left(\tilde{J}_{0 z}+\tilde{J}_{z z}\right)\right), \\
& \frac{d \tilde{J}_{0 x}}{d \ln \Lambda}=\frac{a}{\pi \hbar\left(c+v_{F}\right)}\left(\tilde{x}_{x 0} \tilde{J}_{x x}-\tilde{J}_{x z} \tilde{J}_{y y}+\left(\tilde{J}_{00}+\tilde{J}_{z 0}\right)\right. \\
& \left.\times\left(\tilde{J}_{0 x}+\tilde{J}_{z x}\right)\right), \\
& \frac{d \tilde{J}_{x 0}}{d \ln \Lambda}=\frac{a}{\pi \hbar\left(c+v_{F}\right)}\left(\tilde{J}_{x 0} \tilde{J}_{00}+\tilde{J}_{x 0} \tilde{J}_{z 0}+\tilde{J}_{x x} \tilde{J}_{0 x}+\tilde{J}_{x x} \tilde{J}_{z x}\right. \\
& \left.+\tilde{J}_{x z} \tilde{J}_{0 z}+\tilde{J}_{x z} \tilde{J}_{z z}\right) \text {, } \\
& \frac{d \tilde{J}_{x z}}{d \ln \Lambda}=\frac{a}{\pi \hbar\left(c+v_{F}\right)}\left(\tilde{J}_{x z} \tilde{J}_{00}+\tilde{J}_{x z} \tilde{J}_{z 0}+\tilde{J}_{y y} \tilde{J}_{0 x}+\tilde{J}_{y y} \tilde{J}_{z x}\right. \\
& \left.+\tilde{J}_{x 0} \tilde{J}_{0 z}+\tilde{J}_{x 0} \tilde{J}_{z z}\right),
\end{aligned}
$$

FIG. 6. Density plot of the renormalized backscattering amplitude $h / h_{0}$ as a function of bare spin-spin $(J)$ and Coulomb $\left(J_{00}\right)$ interactions. The spin-orbit couplings at each point of the phase diagram are taken to be $J_{x 0}=J_{0 x}=J_{y 0}=J_{0 y}=0.3\left(J_{00}+J\right)$. The three phases are qualitatively robust with respect to the SO interactions, although the concrete infrared values of $h$ are affected by these couplings.

$$
\begin{aligned}
\frac{d \tilde{J}_{z x}}{d \ln \Lambda}= & \frac{a}{\pi \hbar\left(c+v_{F}\right)}\left(-\tilde{J}_{x 0} \tilde{x}_{x x}+\tilde{J}_{x z} \tilde{J}_{y y}+\left(\tilde{J}_{00}+\tilde{J}_{z 0}\right)\right. \\
& \left.\times\left(\tilde{J}_{0 x}+\tilde{J}_{z x}\right)\right), \\
\frac{d h}{d \ln \Lambda}= & -\frac{a^{2} h}{2 \pi^{2} \hbar^{2} v_{F}\left(c+v_{F}\right)}(\alpha(J)+\alpha(\tilde{J})), \\
\frac{d v_{F}}{d \ln \Lambda}= & -\frac{2 v_{F}}{\pi^{2}\left(c+v_{F}\right)^{2}}\left(J_{x x}^{2}+J_{y y}^{2}+J_{x 0}^{2}+J_{x z}^{2}\right),
\end{aligned}
$$

where we defined

$$
\begin{aligned}
\alpha(J)= & J_{00}^{2}+J_{0 x}^{2}+J_{0 z}^{2}+J_{x 0}^{2}+J_{x x}^{2}+J_{x z}^{2}-J_{y y}^{2}-J_{z 0}^{2}-J_{z x}^{2} \\
& -J_{z z}^{2},
\end{aligned}
$$

and, as before, assumed symmetry between the two channels for the Fermi velocity $v_{F}$ renormalization, which can be consistently done also in the presence of the spin-orbital coupling.

In Fig. 6, plotted for the $\mathrm{Bi}_{2} \mathrm{Te}_{3}$ case, one can see that even in the presence of the $\mathrm{SO}$ interactions all three regimes (enhancement marginal suppression, and strict suppression of the backscattering) survive, and the additional coupling only changes the particular renormalized values of the backscattering amplitude.
[1] C. L. Kane and E. J. Mele, $\mathbb{Z}_{2}$ Topological Order and the Quantum Spin Hall Effect, Phys. Rev. Lett. 95, 146802 (2005).

[2] C. L. Kane and E. J. Mele, Quantum Spin Hall Effect in Graphene, Phys. Rev. Lett. 95, 226801 (2005).
[3] B. A. Bernevig, T. L. Hughes, and S.-C. Zhang, Quantum spin Hall effect and topological phase transition in $\mathrm{HgTe}$ quantum wells, Science 314, 1757 (2006).

[4] M. König, S. Wiedmann, C. Brüne, A. Roth, H. Buhmann, L. W. Molenkamp, X.-L. Qi, and S.-C. Zhang, Quantum spin 
hall insulator state in HgTe quantum wells, Science 318, 766 (2007).

[5] M. Z. Hasan and C. L. Kane, Colloquium: Topological insulators, Rev. Mod. Phys. 82, 3045 (2010).

[6] X.-L. Qi and S.-C. Zhang, Topological insulators and superconductors, Rev. Mod. Phys. 83, 1057 (2011).

[7] B. Keimer and J. E. Moore, The physics of quantum materials, Nat. Phys. 13, 1045 (2017).

[8] Y. Hatsugai, Edge states in the integer quantum Hall effect and the Riemann surface of the Bloch function, Phys. Rev. B 48, 11851 (1993).

[9] Y. Hatsugai, Chern Number and Edge States in the Integer Quantum Hall Effect, Phys. Rev. Lett. 71, 3697 (1993).

[10] E. Prodan, The edge spectrum of Chern insulators with rough boundaries, J. Math. Phys. 50, 083517 (2009).

[11] J.-W. Rhim, J. H. Bardarson, and R.-J. Slager, Unified bulkboundary correspondence for band insulators, Phys. Rev. B 97, 115143 (2018).

[12] N. Kainaris, I. V. Gornyi, S. T. Carr, and A. D. Mirlin, Conductivity of a generic helical liquid, Phys. Rev. B 90, 075118 (2014).

[13] P. D. Kurilovich, V. D. Kurilovich, I. S. Burmistrov, and M. Goldstein, Helical edge transport in the presence of a magnetic impurity, JETP Lett. 106, 593 (2017).

[14] I. S. Burmistrov and E. V. Repin, Quantum corrections to conductivity of disordered electrons due to inelastic scattering off magnetic impurities, Phys. Rev. B 98, 045414 (2018).

[15] T. L. Schmidt, S. Rachel, F. von Oppen, and L. I. Glazman, Inelastic Electron Backscattering in a Generic Helical Edge Channel, Phys. Rev. Lett. 108, 156402 (2012).

[16] M. Hohenadler and F. F. Assaad, Luttinger liquid physics and spin-flip scattering on helical edges, Phys. Rev. B 85, 081106(R) (2012)

[17] M. Hohenadler and F. F. Assaad, Rashba coupling and magnetic order in correlated helical liquids, Phys. Rev. B 90, 245148 (2014)

[18] J. Maciejko, C. Liu, Y. Oreg, X.-L. Qi, C. Wu, and S.-C. Zhang, Kondo Effect in the Helical Edge Liquid of the Quantum Spin Hall State, Phys. Rev. Lett. 102, 256803 (2009).

[19] Y. Tanaka, A. Furusaki, and K. A. Matveev, Conductance of a Helical Edge Liquid Coupled to a Magnetic Impurity, Phys. Rev. Lett. 106, 236402 (2011).

[20] J. I. Väyrynen, M. Goldstein, and L. I. Glazman, Helical Edge Resistance Introduced by Charge Puddles, Phys. Rev. Lett. 110, 216402 (2013).

[21] J. I. Väyrynen, M. Goldstein, Y. Gefen, and L. I. Glazman, Resistance of helical edges formed in a semiconductor heterostructure, Phys. Rev. B 90, 115309 (2014).

[22] P. Novelli, F. Taddei, A. K. Geim, and M. Polini, Failure of Conductance Quantization in Two-Dimensional Topological Insulators due to Nonmagnetic Impurities, Phys. Rev. Lett. 122, 016601 (2019).

[23] J. Moore, Topological insulators: The next generation, Nat. Phys. 5, 378 (2009).
[24] W. H. Zurek, Decoherence, einselection, and the quantum origins of the classical, Rev. Mod. Phys. 75, 715 (2003).

[25] E. Joos, H. D. Zeh, C. Kiefer, D. J. W. Giulini, J. Kupsch, and I. O. Stamatescu, Decoherence and the Appearance of a Classical World in Quantum Theory (Springer, Berlin, 2003).

[26] H. C. Donker, H. De Raedt, and M. I. Katsnelson, Decoherence and pointer states in small antiferromagnets: A benchmark test, SciPost Phys. 2, 010 (2017).

[27] A. O. Caldeira and A. J. Leggett, Quantum tunneling in a dissipative system, Ann. Phys. (NY) 149, 374 (1983).

[28] A. J. Leggett, S. Chakravarty, A. T. Dorsey, Matthew P. A. Fisher, Anupam Garg, and W. Zwerger, Dynamics of the dissipative two-state system, Rev. Mod. Phys. 59, 1 (1987).

[29] N. V. Prokof'ev and P. C. E. Stamp, Theory of the spin bath, Rep. Prog. Phys. 63, 669 (2000).

[30] C.-H. Hsu, P. Stano, J. Klinovaja, and D. Loss, Nuclear-spininduced localization of edge states in two-dimensional topological insulators, Phys. Rev. B 96, 081405(R) (2017)

[31] C.-H. Hsu, P. Stano, J. Klinovaja, and D. Loss, Effects of nuclear spins on the transport properties of the edge of twodimensional topological insulators, Phys. Rev. B 97, 125432 (2018).

[32] A. Abragam, The Principles of Nuclear Magnetism (Clarendon, Oxford, 1961).

[33] C. P. Slichter, The Principles of Magnetic Resonance (SpringerVerlag, Berlin, 1990).

[34] M. A. Cazalilla, F. Sols, and F. Guinea, Dissipation-Driven Quantum Phase Transitions in a Tomonaga-Luttinger Liquid Electrosatically Coupled to a Metallic Gate, Phys. Rev. Lett. 97, 076401 (2006).

[35] J. Appel, Polarons, in Solid State Physics, edited by H. Ehrenreich, F. Seitz, and D. Turnbull (Academic, New York, 1968), Vol. 21.

[36] G. D. Mahan, Many-Particle Physics (Kluwer Academic, Plenum, New York, 2000).

[37] P. W. Anderson, A poor man's derivation of scaling laws for the Kondo problem, J. Phys. C 3, 2436 (1970).

[38] P. Nozieres and A. Blandin, Kondo effect in real metals, J. Phys. France 41, 193 (1980).

[39] V. Yu. Irkhin and M. I. Katsnelson, Interaction of conduction electrons with local excitations. The infrared divergencies, Z. Phys. B 70, 371 (1988).

[40] V. Yu. Irkhin and M. I. Katsnelson, Scaling picture of magnetism formation in the anomalous f-electron systems: Interplay of the Kondo effect and spin dynamics, Phys. Rev. B 56, 8109 (1997).

[41] D.-X. Qu, Y. S. Hor, Jun Xiong, R. J. Cava, and N. P. Ong, Quantum oscillations and Hall anomaly of surface states in the topological insulator $\mathrm{Bi}_{2} \mathrm{Te}_{3}$, Science 329, 821 (2010).

[42] X.-G. Zhu, Y. Zhang, W. Feng, B.-K. Yuan, Q. Liu, R.-Z. Qiu, D.-H. Xie, S.-Y. Tan, Y. Duan, Y. Fang et al., Electronic structures of topological insulator $\mathrm{Bi}_{2} \mathrm{Te}_{3}$ surfaces with nonconventional terminations, New J. Phys. 18, 093015 (2016). 